\title{
MCIT \\ Spatial Analogues of Numerical Quasiconformal Mapping Methods for Solving Problems of Bursts Parameters Identification
}

https://doi.org/10.31713/MCIT.2020.10

\author{
Mykhailo Boichura \\ Research dept. \\ National University of Water and Environmental Engineering \\ Rivne, Ukraine \\ m.v.boichura@nuwm.edu.ua
}

\begin{abstract}
An approach to solving the problem of image reconstruction based on applied quasipotential tomographic data in the three-dimensional case is developed. It is based on the synthesis of spatial analogues of numerical quasiconformal mapping methods and algorithm for identifying the parameters of local bursts of homogeneous materials using similar methods on the plane. The peculiarity of the corresponding algorithm is taking into account (for each of the appropriate injections) the presence of only equipotential lines (with given values of the flow function or distributions of local velocities on them) and flow lines (with known potential distributions on them) at the domain boundary. Numerical experiments of simulative restoration of the environment structure are carried out.
\end{abstract}

Keywords-applied quasipotential tomography; quasiconformal mappings; identification; numerical methods; impedance tomography

\section{INTRODUCTION}

A huge number of works are devoted to the development of means for image reconstruction of the structure of closed media without appropriate physical intervention $[1,2]$. However, many non-invasive methods, nevertheless, are harmful to the object. For example, most of these medical imaging methods have a direct negative impact on the internal organs. Some are expensive, not suitable for observing the object in dynamics, etc.

This paper proposes one of the approaches to image reconstruction using impedance tomography methods. Its advantages include the following: non-harmful to health, low cost (in maintenance and direct application), flexibility, ease of use, the possibilities of observation in dynamics, calculation numerical (non-analog) characteristics and research a wide class of objects, etc. [2]. A significant disadvantage of impedance diagnostics is the low quality of the obtained images. A number of scientists work to solve this problem [1, 2]. However, in practice, the distributions of current densities along the potential application areas are taken as the average values [1,2], which, of course, negatively affects the accuracy of the reconstruction.
Successful attempts to avoid the "pointiness" of the quasipotential application areas were carried out in [3 - 5] with using the numerical quasiconformal mapping methods for modeling current density fields. In particular, in [4], an algorithm for solving the problem of impedance tomography in the case of setting the structure of the conductivity coefficient (CC) in the form of a function of local bursts of homogeneous materials is proposed. We propose to extend these ideas to the space and thereby making the approach proposed in [4] one step closer to practice. In this case, the CC [4] is supplemented by the $\left(z-z_{k}\right)^{2}$ term as follows:

$$
\begin{gathered}
\sigma\left(x, y, z, \xi, \alpha_{1}, \varepsilon_{1}, x_{1}, y_{1}, z_{1}, \ldots, \alpha_{s}, \varepsilon_{s}, x_{s}, y_{s}, z_{s}\right)=\xi+ \\
+\sum_{k=1}^{s} \frac{\alpha_{k}}{1+\left(\eta_{k}\left(x-x_{k}\right)^{2}+\mu_{k}\left(y-y_{k}\right)^{2}+\lambda_{k}\left(z-z_{k}\right)^{2}\right) / \varepsilon_{k}},
\end{gathered}
$$

where $\xi, \alpha_{k}, \varepsilon_{k}, x_{k}, y_{k}, z_{k}(k=1, \ldots, s)$ are the parameters sought during the process of problem solving, $\eta_{k}, \mu_{k}, \lambda_{k}$ are given constants. We propose to take the main ideas for the construction of spatial formulation of the impedance tomography problem and corresponding algorithm for its solution from $[5,6]$.

\section{Statement of the Impedance Tomography Problem}

Suppose that quasiideal processes of particle (fluid or charge) movement occur due to the actions of applied quasipotentials $\varphi_{*}^{(p)}$ and $\varphi^{*(p)} \quad\left(\varphi^{*(p)}>\varphi_{*}^{(p)}\right)$ differences between the $A_{*_{p}} B_{*_{p}} B_{p}^{*} A_{p}^{*}$ and $C_{*_{p}} D_{*_{p}} D_{p}^{*} C_{p}^{*}$ sections at the body (rod i.e. tomographic object) $G_{\tau}$ (fig. 1,a). Each such situational case, configured by fixing the eight marked points $A_{*}, A_{p}^{*}$, $B_{* p}, \quad B_{p}^{*}, \quad C_{*_{p}}, \quad C_{p}^{*}, \quad D_{*_{p}}, \quad D_{p}^{*}$ on the $\partial G_{\tau}=\{\tau=(x, y, z)$ : $\tau=(\tilde{x}(u, v), \tilde{y}(u, v), \tilde{z}(u, v)), 0 \leq u \leq 1,0 \leq v \leq 1\}$ boundary, 


\section{Modeling, control and information technologies -2020}

we call injection (see, e.g. [2-5]); for convenience, we denote $G_{\tau}$ and $\partial G_{\tau}$ by $G_{\tau}^{(p)}$ and $\partial G_{\tau}^{(p)}$, respectively. Here $p=1,2, \ldots$ is injection number; $A_{*_{p}} B_{*_{p}} C_{*_{p}} D_{*_{p}}, B_{*_{p}} C_{*_{p}} C_{p}^{*} B_{p}^{*}$, $A_{* p} D_{*_{p}} D_{p}^{*} A_{p}^{*}$ and $A_{p}^{*} B_{p}^{*} C_{p}^{*} D_{p}^{*}$ are impermeable flow boundary surfaces; $A_{*_{p}} B_{*_{p}} B_{p}^{*} A_{p}^{*}$ and $C_{*_{p}} D_{*_{p}} D_{p}^{*} C_{p}^{*}$ are equipotential surfaces; $\tilde{x}(u, v), \tilde{y}(u, v), \quad \tilde{z}(u, v)$ are defined continuously differentiable functions.

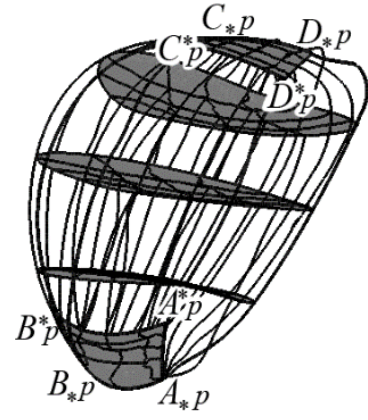

a)

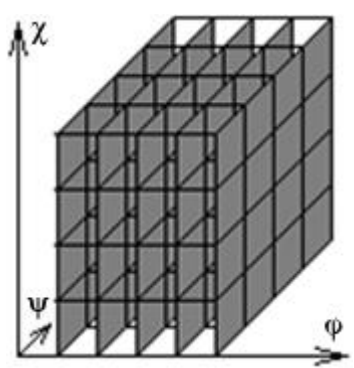

b)
Figure 1. Tomographic body $G_{\tau}$ (a) and corresponding complex quasipotential domains $G_{\omega}^{(p)}$ (b)

In most cases, the image reconstruction using applied quasipotential tomographic data is carried out by alternating iterative solving of a number of sub problems [ $1-5]$ of modeling of current density fields (in this case, the functions of quasipotential $\quad \varphi^{(p)}=\varphi^{(p)}(x, y, z)$ and currents $\psi^{(p)}=\psi^{(p)}(x, y, z), \quad \chi^{(p)}=\chi^{(p)}(x, y, z)$ are also sought) and identification of CC $\sigma=\sigma(x, y, z)$ parameters. We, similarly to [6], propose to write the statement of the first of these (with a given structure of $\mathrm{CC}(1))$ in the form:

$$
\begin{aligned}
& \left\{\begin{array}{l}
\sigma(x, y, z) \operatorname{grad} \varphi^{(p)}(x, y, z)= \\
=\operatorname{grad} \psi^{(p)}(x, y, z) \times \operatorname{grad} \chi^{(p)}(x, y, z), \\
\operatorname{grad} \psi^{(p)}(x, y, z) \operatorname{grad} \chi^{(p)}(x, y, z)=0, \tau \in \tilde{G}_{\tau}^{(p)} ;
\end{array}\right. \\
& \left.\varphi^{(p)}\right|_{A_{* p} B_{* p} B_{p}^{*} A_{p}^{*}}=\varphi_{*}^{(p)},\left.\quad \varphi^{(p)}\right|_{C_{* p} D_{* p} D_{p}^{*} C_{p}^{*}}=\varphi^{*(p)}, \\
& \left.\psi^{(p)}\right|_{A_{* p} D_{* p} D_{p}^{*} A_{p}^{*}}=0,\left.\quad \psi^{(p)}\right|_{B_{* p} C_{* p} C_{p}^{*} B_{p}^{*}}=\bar{Q}^{(p)}, \\
& \left.\chi^{(p)}\right|_{A_{* p} B_{* p} C_{* p} D_{* p}}=0,\left.\quad \chi^{(p)}\right|_{A_{p}^{*} B_{p}^{*} C_{p}^{*} D_{p}^{*}}=\widehat{Q}^{(p)} ; \\
& \bar{Q}^{(p)} \widehat{Q}^{(p)}=\int_{A_{*} B_{* p}} \sigma \frac{\partial \varphi^{(p)}}{\partial n} d s \int_{A_{* p} A_{p}^{*}} \sigma \frac{\partial \varphi^{(p)}}{\partial n} d s=Q^{(p)},
\end{aligned}
$$

where $p=\overline{1, \tilde{p}} ; \quad \vec{n}$ is a unit normal vector; $\bar{Q}^{(p)}, \widehat{Q}^{(p)}$ are the redistributions of discharges $Q^{(p)}$ of vector fields (the magnitudes of the current) along the horizontal and vertical unit layers of arbitrary cross sections, respectively [6]. While the sub problem of CC identification (1), like in [5], we propose to solve using refined parameters of the current density fields (as a solution of sub problem (1) - (3)) and under additional conditions

$$
\begin{aligned}
& \left.\varphi^{(p)}(M)\right|_{A_{* p} D_{* p} D_{p}^{*} A_{p}^{*}}=\underline{\varphi}^{(p)}(M), \\
& \left.\varphi^{(p)}(M)\right|_{B_{* p} C_{* p} C_{p}^{*} B_{p}^{*}}=\bar{\varphi}^{(p)}(M), \\
& \left.\chi^{(p)}(M)\right|_{A_{* p} D_{* p} D_{p}^{*} A_{p}^{*}}=\underline{\underline{\chi}}^{(p)}(M), \\
& \left.\chi^{(p)}(M)\right|_{B_{* p} C_{* p} C_{p}^{*} B_{p}^{*}}=\overline{\bar{\chi}}^{(p)}(M), \\
& \left.\psi^{(p)}(M)\right|_{A_{* p} B_{* p} B_{p}^{*} A_{p}^{*}}=\psi_{* *}^{(p)}(M), \\
& \left.\psi^{(p)}(M)\right|_{C_{* p} D_{* p} D_{p}^{*} C_{p}^{*}}=\psi^{* *(p)}(M), \\
& \left.\chi^{(p)}(M)\right|_{A_{* p} B_{* p} B_{p}^{*} A_{p}^{*}}=\chi_{* *}^{(p)}(M), \\
& \left.\chi^{(p)}(M)\right|_{C_{* p} D_{* p} D_{p}^{*} C_{p}^{*}}=\chi^{* *(p)}(M), \\
& \left.\varphi^{(p)}(M)\right|_{A_{* p} B_{* p} C_{* p} D_{*} p}=\varphi^{(p)}(M), \\
& \left.\varphi^{(p)}(M)\right|_{A_{p}^{*} B_{p}^{*} C_{p}^{*} D_{p}^{*}}=\hat{\varphi}^{(p)}(M), \\
& \left.\psi^{(p)}(M)\right|_{A_{* p} B_{* p} C_{* p} D_{* p}}=\psi^{(p)}(M), \\
& \left.\psi^{(p)}(M)\right|_{A_{p}^{*} B_{p}^{*} C_{p}^{*} D_{p}^{*}}=\widehat{\widehat{\psi}}^{(p)}(M) .
\end{aligned}
$$

Here $p=\overline{1, \tilde{p}} ; \quad M$ is a running point on the corresponding curve;

$$
\begin{aligned}
& G_{\omega}^{(p)}=\left\{\omega=(\varphi, \psi, \chi): \quad \varphi_{*}^{(p)} \leq \varphi \leq \varphi^{*(p)}, \quad 0 \leq \psi \leq \bar{Q}^{(p)},\right. \\
& \left.0 \leq \chi \leq \widehat{Q}^{(p)}\right\} ; \quad \psi_{* *}^{(p)}(M)=\int_{A_{*} p M} \psi_{*}^{(p)}(M) d s, \\
& \psi^{\psi^{(p)}}(M)=\int_{A_{* p} M} \psi^{(p)}(M) d s, \quad \psi^{* *(p)}(M)=\int_{D_{*} p} \psi^{*(p)}(M) d s, \\
& \widehat{\bar{\psi}}^{(p)}(M)=\int_{A_{p}^{*} M} \widehat{\psi}^{(p)}(M) d s, \quad \chi_{* *}^{(p)}(M)=\int_{A_{*} p} \chi_{*}^{(p)}(M) d s, \\
& \underline{\underline{\chi}}^{(p)}(M)=\int_{A_{* p} M} \underline{\chi}^{(p)}(M) d s, \quad \chi^{* *(p)}(M)=\int_{D_{* p} M} \chi^{*(p)}(M) d s, \\
& \overline{\bar{\chi}}^{(p)}(M)=\int_{B_{* p} M} \bar{\chi}^{(p)}(M) d s ; \underline{\varphi}^{(p)}(M), \underline{\varphi}^{(p)}(M), \bar{\varphi}^{(p)}(M), \\
& \hat{\varphi}^{(p)}(M), \quad \underline{\chi}^{(p)}(M), \quad \bar{\chi}^{(p)}(M), \quad \psi_{*}^{(p)}(M), \quad \psi^{*(p)}(M), \\
& \chi_{*}^{(p)}(M), \quad \chi^{*(p)}(M), \quad \psi^{(p)}(M), \quad \widehat{\psi}^{(p)}(M) \text { are functions }
\end{aligned}
$$
$\left(u_{i^{\prime}}^{*(p)}, v_{i^{\prime}}^{*(p)}\right),\left(\bar{u}_{i^{\prime}}^{(p)}, \bar{v}_{i^{\prime}}^{(p)}\right)$ at the corresponding sections; 
Modeling, control and information technologies $\mathbf{- 2 0 2 0}$

$$
\begin{gathered}
\varphi_{*}^{(p)} \leq \underline{\varphi}_{i^{\prime}}^{(p)},{\underline{i^{\prime}}}^{(p)}, \bar{\varphi}_{i^{\prime}}^{(p)}, \hat{\varphi}_{i^{\prime}}^{(p)} \leq \varphi^{*(p)} ; \\
0 \leq \psi_{* * i^{\prime}}^{(p)}, \underbrace{(p)}_{i^{\prime}}, \psi_{i^{\prime}}^{* *(p)}, \widehat{\bar{\psi}}_{i^{\prime}}^{(p)} \leq \bar{Q}^{(p)} ; \\
\psi_{* i^{\prime}}^{(p)}, \psi_{i^{\prime}}^{(p)}, \psi_{i^{\prime}}^{*(p)}, \widehat{\psi}_{i^{\prime}}^{(p)}, \chi_{i_{i^{\prime}}}^{(p)},{\underline{\chi_{i}}}_{i^{\prime}}^{(p)}, \chi_{i^{\prime}}^{*(p)}, \bar{\chi}_{i^{\prime}}^{(p)}>0 ; \\
0 \leq \chi_{* * i^{\prime}}^{(p)}, \underline{\chi}_{i^{\prime}}^{(p)}, \chi_{i^{\prime}}^{* *(p)}, \overline{\bar{\chi}}_{i^{\prime}}^{(p)} \leq \widehat{Q}^{(p)} ;
\end{gathered}
$$

discharges $\bar{Q}^{(p)}, \quad \widehat{Q}^{(p)}$ are obtained as a result of physical measurements too.

\section{SYNTHESIS OF THE NUMERICAL QUASICONFORMAL MAPPING METHOD FOR SOLVING THE IMPEDANCE TOMOGRAPHY PROBLEM}

The complexity of solving the problem (2) - (4) when functions $\quad \varphi^{(p)}=\varphi^{(p)}(x, y, z), \quad \psi^{(p)}=\psi^{(p)}(x, y, z)$, $\chi^{(p)}=\chi^{(p)}(x, y, z)$ and $\sigma=\sigma(x, y, z)$ are sought arises, first of all, due to irregularity of spatial meshes, which correspond to fig. 1,a. A solution to this problem is given in [6, 7]. Namely: it is proposed to carry out the corresponding inverse quasiconformal mappings of complex quasipotential domains (with regular meshes) $G_{\omega}^{(p)}$ on the corresponding curvilinear physical domains $G_{z}^{(p)}$ with the sought $x^{(p)}=x^{(p)}(\varphi, \psi, \chi)$, $y^{(p)}=y^{(p)}(\varphi, \psi, \chi), \quad z^{(p)}=z^{(p)}(\varphi, \psi, \chi)$ and $\sigma=\sigma(x, y, z)$ $(p=\overline{1, \tilde{p}})$ :

$$
\begin{aligned}
& \int\left(\sigma^{-1} x_{\varphi}^{\prime(p)}\right)_{\varphi}^{\prime}+\left(\sigma x_{\psi}^{\prime(p)} / \mathrm{X}^{(p) 2}\right)_{\psi}^{\prime}+\left(\sigma x_{\chi}^{\prime(p)} / \Psi^{(p) 2}\right)_{\chi}^{\prime}=0, \\
& \left\{\left(\sigma^{-1} y_{\varphi}^{\prime(p)}\right)_{\varphi}^{\prime}+\left(\sigma y_{\psi}^{(p)} / \mathrm{X}^{(p) 2}\right)_{\psi}^{\prime}+\left(\sigma y_{\chi}^{\prime(p)} / \Psi^{(p) 2}\right)_{\chi}^{\prime}=0,\right. \\
& \left(\sigma^{-1} z_{\varphi}^{\prime(p)}\right)_{\varphi}^{\prime}+\left(\sigma z_{\psi}^{(p)} / \mathrm{X}^{(p) 2}\right)_{\psi}^{\prime}+\left(\sigma z_{\chi}^{\prime(p)} / \Psi^{(p) 2}\right)_{\chi}^{\prime}=0 ; \\
& \left.\tau^{(p)}\left(\varphi_{*}^{(p)}, \psi, \chi\right)\right|_{A_{*_{p}} B_{*} B_{p}^{*} A_{p}^{*}}=\tilde{r}\left(u_{*}^{(p)}(\psi, \chi), v_{*}^{(p)}(\psi, \chi)\right), \\
& \left.\tau^{(p)}\left(\varphi^{*(p)}, \psi, \chi\right)\right|_{C_{*_{p}} D_{*_{p}} D_{p}^{*} C_{p}^{*}}=\tilde{r}\left(u^{*(p)}(\psi, \chi), v^{*(p)}(\psi, \chi)\right), \\
& \left.\tau^{(p)}(\varphi, 0, \chi)\right|_{A_{*_{p}} D_{* p} D_{p}^{*} A_{p}^{*}}=\tilde{r}\left(\underline{u}^{(p)}(\varphi, \chi), \underline{v}^{(p)}(\varphi, \chi)\right), \\
& \left.\tau^{(p)}\left(\varphi, \bar{Q}^{(p)}, \chi\right)\right|_{B_{*_{p}} C_{*_{p}} C_{p}^{*} B_{p}^{*}}=\tilde{r}\left(\bar{u}^{(p)}(\varphi, \chi), \bar{v}^{(p)}(\varphi, \chi)\right),
\end{aligned}
$$

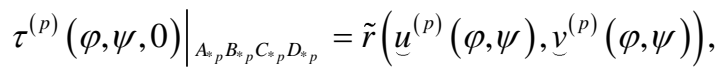

$$
\begin{aligned}
& \left.\tau^{(p)}\left(\varphi, \psi, \widehat{Q}^{(p)}\right)\right|_{A_{p}^{*} B_{p}^{*} C_{p}^{*} D_{p}^{*}}=\tilde{r}\left(\widehat{u}^{(p)}(\varphi, \psi), \widehat{v}^{(p)}(\varphi, \psi)\right)
\end{aligned}
$$

provided that the conditions [5, 6] of orthogonality at the boundary are fulfilled, where $\tilde{r}(u, v)=(\tilde{x}(u, v), \tilde{y}(u, v), \tilde{z}(u, v)) ; \quad \mathrm{X}=\mathrm{X}(x, y, z) ;$ $\Psi=\Psi(x, y, z) ; \quad J=D(\tau) / D(\omega)$ is the Jacobian transformation; $\quad u=u_{*}^{(p)}(\psi, \chi), \quad v=v_{*}^{(p)}(\psi, \chi)$, $u=\underline{u}^{(p)}(\varphi, \chi), \quad v=\underline{v}^{(p)}(\varphi, \chi), \quad u=\underline{u}^{(p)}(\varphi, \psi)$, $v=v^{(p)}(\varphi, \psi)$,

$u=u^{*(p)}(\psi, \chi)$, $v=v^{*(p)}(\psi, \chi)$,

$u=\bar{u}^{(p)}(\varphi, \chi)$,

$v=\bar{v}^{(p)}(\varphi, \chi)$, $u=\widehat{u}^{(p)}(\varphi, \psi)$, $v=\widehat{v}^{(p)}(\varphi, \psi) \quad\left(\varphi_{*}^{(p)} \leq \varphi \leq \varphi^{*(p)}, \quad 0 \leq \psi \leq \bar{Q}^{(p)}, \quad 0 \leq \chi \leq \widehat{Q}^{(p)}\right)$ are functions constructed by interpolation of their experimentally obtained values $u_{*_{i^{\prime}}^{\prime}}^{(p)}, \quad v_{*_{i^{\prime}}}^{(p)}, \quad \underline{u}_{i^{\prime}}^{(p)}, \quad \underline{v}_{i^{\prime}}^{(p)}, \quad \underline{u}_{i^{\prime}}^{(p)}, \quad v_{i^{\prime}}^{(p)}, \quad u_{i^{\prime}}^{*(p)}$, $v_{i^{\prime}}^{*(p)}, \quad \bar{u}_{i^{\prime}}^{(p)}, \quad \bar{v}_{i^{\prime}}^{(p)}, \quad \widehat{u}_{i^{\prime}}^{(p)}, \quad \widehat{v}_{i^{\prime}}^{(p)}$ having arguments $\psi_{* *}^{(p)}\left(u_{* i^{\prime}}^{(p)}, v_{* i^{\prime}}^{(p)}\right), \quad \chi_{* *}^{(p)}\left(u_{* i^{\prime}}^{(p)}, v_{* i^{\prime}}^{(p)}\right), \underline{\varphi}_{i^{\prime}}^{(p)}, \underline{\underline{\chi}}^{(p)}\left(\underline{u}_{i^{\prime}}^{(p)}, \underline{v}_{i^{\prime}}^{(p)}\right), \underline{\varphi}_{i^{\prime}}^{(p)}$, $\psi^{(p)}\left(u_{i^{\prime}}^{(p)}, v_{i^{\prime}}^{(p)}\right), \quad \psi^{* *(p)}\left(u_{i^{\prime}}^{*(p)}, v_{i^{\prime}}^{*(p)}\right), \quad \chi^{* *(p)}\left(u_{i^{\prime}}^{*(p)}, v_{i^{\prime}}^{*(p)}\right), \bar{\varphi}_{i^{\prime}}^{(p)}$, $\overline{\bar{\chi}}^{(p)}\left(\bar{u}_{i^{\prime}}^{(p)}, \bar{v}_{i^{\prime}}^{(p)}\right), \quad \widehat{\varphi}_{i^{\prime}}^{(p)}, \quad \widehat{\hat{\psi}}^{(p)}\left(\widehat{u}_{i^{\prime}}^{(p)}, \widehat{v}_{i^{\prime}}^{(p)}\right)$ at the corresponding sections; $\mathrm{X}^{(p)}=\mathrm{X}\left(x^{(p)}, y^{(p)}, z^{(p)}\right), \quad \Psi^{(p)}=\Psi\left(x^{(p)}, y^{(p)}, z^{(p)}\right)$, $\mathrm{X}=\sqrt{\left(y_{\varphi} z_{\psi}-z_{\varphi} y_{\psi}\right)^{2}+\left(z_{\varphi} x_{\psi}-x_{\varphi} z_{\psi}\right)^{2}+\left(x_{\varphi} y_{\psi}-y_{\varphi} x_{\psi}\right)^{2}} / J$, $\Psi=\sqrt{\left(y_{\chi} z_{\varphi}-z_{\chi} y_{\varphi}\right)^{2}+\left(z_{\chi} x_{\varphi}-x_{\chi} z_{\varphi}\right)^{2}+\left(x_{\chi} y_{\varphi}-y_{\chi} x_{\varphi}\right)^{2}} / J$.

We will reconstruct the $\mathrm{CC}$, similarly to $[4,5]$, by providing a minimum of functional

$$
\Phi\left(\tau^{(1)}, \ldots, \tau^{(\tilde{p})}, \sigma\right)=\sum_{p=1}^{\tilde{p}}\left(\sigma\left(\tau^{(p)}, \ldots\right)-f\left(\tau^{(p)}\right)\right)^{2} \rightarrow \min
$$

with respect to the desired $\xi, \alpha_{k}, \varepsilon_{k}, x_{k}, y_{k}(k=1, \ldots, s)$, within the $\sigma\left(\tau^{(p)}, \ldots\right)=\sigma\left(\tau^{(p)}, \xi, \alpha_{1}, \varepsilon_{1}, x_{1}, y_{1}, z_{1}, \ldots, \alpha_{s}, \varepsilon_{s}, x_{s}, y_{s}, z_{s}\right)$,

where

$$
f\left(\tau^{(p)}\right)=\frac{\partial \tau^{(p)}}{\partial \varphi} /\left(\frac{\partial \tau^{(p)}}{\partial \psi} \frac{\partial \tau^{(p)}}{\partial \chi}\right) .
$$

In the general case of input data, the differential problem of impedance tomography is replaced by the corresponding difference $[1,2,5,6]$. The latter is solved with respect to the nodal points $\quad x_{i, j, k}^{(p)}=x^{(p)}\left(\varphi_{i}^{(p)}, \psi_{j}^{(p)}, \chi_{k}^{(p)}\right)$, $y_{i, j, k}^{(p)}=y^{(p)}\left(\varphi_{i}^{(p)}, \psi_{j}^{(p)}, \chi_{k}^{(p)}\right), \quad z_{i, j, k}^{(p)}=z^{(p)}\left(\varphi_{i}^{(p)}, \psi_{j}^{(p)}, \chi_{k}^{(p)}\right)$ and parameters $\chi, \alpha_{1}, \varepsilon_{1}, x_{1}, y_{1}, z_{1}, \ldots, \alpha_{s}, \varepsilon_{s}, x_{s}, y_{s}, z_{s}$ of CC (1). The difference representation of this problem has a form similar to $[5,6]$. In this case, we write the right part of expression (6) for reasons of maximum consideration of the configurations of both internal and boundary mesh surfaces in the form:

$$
\begin{gathered}
4\left(\rho\left(\tau_{i+1, j, k}^{(p)}, \tau_{i, j, k}^{(p)}\right)+\rho\left(\tau_{i+1, j+1, k}^{(p)}, \tau_{i, j+1, k}^{(p)}\right)+\right. \\
\left.+\rho\left(\tau_{i+1, j, k+1}^{(p)}, \tau_{i, j, k+1}^{(p)}\right)+\rho\left(\tau_{i+1, j+1, k+1}^{(p)}, \tau_{i, j+1, k+1}^{(p)}\right)\right) / \\
/\left(\rho\left(\tau_{i, j+1, k}^{(p)}, \tau_{i, j, k}^{(p)}\right)+\rho\left(\tau_{i+1, j+1, k}^{(p)}, \tau_{i+1, j, k}^{(p)}\right)+\right. \\
\left.+\rho\left(\tau_{i, j+1, k+1}^{(p)}, \tau_{i, j, k+1}^{(p)}\right)+\rho\left(\tau_{i+1, j+1, k+1}^{(p)}, \tau_{i+1, j, k+1}^{(p)}\right)\right) / \\
/\left(\rho\left(\tau_{i, j, k+1}^{(p)}, \tau_{i, j, k}^{(p)}\right)+\rho\left(\tau_{i+1, j, k+1}^{(p)}, \tau_{i+1, j, k}^{(p)}\right)+\right.
\end{gathered}
$$




\section{Modeling, control and information technologies -2020}

$$
\begin{gathered}
\left.+\rho\left(\tau_{i, j+1, k+1}^{(p)}, \tau_{i, j+1, k}^{(p)}\right)+\rho\left(\tau_{i+1, j+1, k+1}^{(p)}, \tau_{i+1, j+1, k}^{(p)}\right)\right) / \gamma^{(p)} ; \\
\rho\left(\tau_{i, j, k}^{(p)}, \tau_{i^{\prime}, j^{\prime}, k^{\prime}}^{(p)}\right)=\left(\left(x_{i, j, k}^{(p)}-x_{i^{\prime}, j^{\prime}, k^{\prime}}^{(p)}\right)^{2}+\right. \\
\left.+\left(y_{i, j, k}^{(p)}-y_{i^{\prime}, j^{\prime}, k^{\prime}}^{(p)}\right)^{2}+\left(z_{i, j, k}^{(p)}-z_{i^{\prime}, j^{\prime}, k^{\prime}}^{(p)}\right)^{2}\right)^{0.5},
\end{gathered}
$$

where $\gamma^{(p)}$ are quasiconformal invariants [6, 7] for the corresponding domains.

The iterative algorithm for solving the problem (1) - (4) by providing a minimum of functional $(5)$ is similar to $[4,5]$ and is based on the alternate parameterization of internal nodes of the mesh domains $G_{\tau}^{\gamma(p)}, \mathrm{CC}$ and use of block iteration methods. A significant difference between the developed approach and [5] is the presence of a large number of extremum points of the minimizing functional (5) due to the nonlinearity of CC (1) with respect to unknown parameters. The corresponding solving process requires the use of global optimization methods (e.g., [8]). In comparison with [4], the algorithm involves a huge amount of similar calculations, which is why it is advisable to use parallelization procedures, which provides, e.g., the CUDA platform [9].

\section{NUMERICAL EXPERIMENTS}

We present the results of numerical experiments of simulative restoration of the medium structure in the case of a plane. Namely: when $\underline{\varphi}_{i^{\prime}}^{(p)}, \bar{\varphi}_{i^{\prime}}^{(p)}, \underline{\chi}_{i^{\prime}}^{(p)}, \bar{\chi}_{i^{\prime}}^{(p)}, \psi_{*^{\prime} i^{\prime}}^{(p)}, \psi_{i^{\prime}}^{(p)}$, $\chi_{* i^{\prime}}^{(p)}, \quad \chi_{i^{\prime}}^{*(p)}, \quad \varphi_{i^{\prime}}^{(p)}, \quad \hat{\varphi}_{i^{\prime}}^{(p)}, \quad \psi_{i^{\prime}}^{(p)}, \quad \widehat{\psi}_{i^{\prime}}^{(p)}$ at the corresponding points and $m^{(p)}, n^{(p)}, l^{(p)}, Q^{(p)}(1 \leq p \leq \tilde{p})$ are given in a tabular form, and $\tilde{x}(u, v)=150 \cos u, \quad \tilde{y}(u, v)=100 \sin u$, $\tilde{z}(u, v)=v \quad(0 \leq u<2 \pi,-30 \leq v \leq 30), \quad \tilde{p}=20, \quad s=2$, $\xi^{(0)}=1, \quad \alpha_{k}^{(0)}=0, \quad \varepsilon_{k}^{(0)}=1, \quad x_{k}^{(0)}=0, \quad y_{k}^{(0)}=0, \quad z_{k}^{(0)}=0$, $\eta_{k}=1, \quad \mu_{k}=1, \quad \lambda_{k}=0, \quad 0.1 \leq \xi \leq 1.5, \quad-2 \leq \alpha_{k} \leq 2$, $10^{-3} \leq \varepsilon_{k} \leq 10^{5}, \quad-150 \leq x_{k} \leq 150, \quad-100 \leq y_{k} \leq 100$, $-30 \leq z_{k} \leq 30(k=1, \ldots, s), \quad \varphi_{*}^{(p)}=0, \varphi^{*(p)}=1 \quad(1 \leq p \leq \tilde{p})$, a reconstructed image of the CC distribution (fig. 2,b) is obtained in comparison with the theoretically known (fig. 2,a).

\section{CONCLUSIONS}

The method of identifying the parameters of the conductivity coefficient, given in the form of a function of local bursts of homogeneous materials using applied quasipotential tomographic data is transferred from the plane to space. Here, on the one hand, the method of image reconstruction, which provides sufficient openness (for various additions, generalizations, etc.), flexibility (for mathematical manipulations), accuracy (because, unlike common practical applications, sections of potential application are considered "non-pointiness") of the corresponding algorithm and also avoidance resource-intensive component of regularization (at least because the method needs no apply of matrix inversion operations) is used. On the other hand, the mathematical formulation and algorithm for solving the problem of modeling a quasiideal flow in a curvilinear parallelepiped bounded by equipotential surfaces and flow surfaces, which is characterized by sufficient stability, convergence and informativeness of results (in comparison with similar methods) are used. The algorithm formed in such way takes into account all the above advantages.

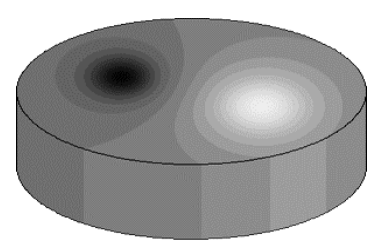

a)

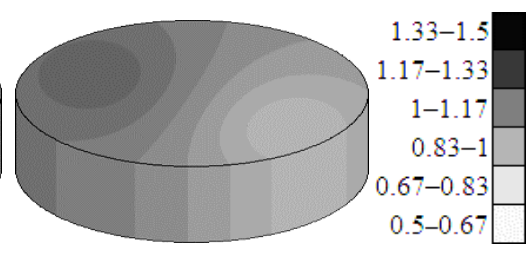

b)
Figure 2. CC distribution: exact (when $\xi=1, \alpha_{1}=-0.5, \quad \varepsilon_{1}=1000$, $x_{1}=60, y_{1}=20, z_{1}=0, \alpha_{2}=0.5, \varepsilon_{2}=400, x_{2}=-60, y_{2}=-20$, $z_{2}=0$ ) (a) and approximated (b) solutions

\section{REFERENCES}

[1] T. K. Bera, "Applications of electrical impedance tomography (EIT): A short review," in IOP Conference Series: Materials Science and Engineering: Proceedings of the 3rd International Conference on Communication Systems: ICCS 2017, October 14-16, 2017, Rajasthan, India, Santosh Jangid, S K Rai Manoj Pandey, Shridhar B Dandin, L Solanki and P S Bhatnagar, Eds. Bristol: Institute of Physics. Vol. 331, pp. 1-8, 2018.

[2] D. Holder, "Electrical Impedance Tomography. Methods, History and Applications," London: Institute of Physics, 2005.

[3] A. Ya. Bomba and L. L. Kroka, "Numerical methods of quasiconformal mappings for solving problems of identifying of electrical conductivity coefficient in an applied potential tomography," Volyn Mathematical Bulletin. Applied Mathematics series, vol. 11 (20), pp. 24-33, 2014 (in Ukrainian).

[4] A. Ya. Bomba and M. V. Boichura, "On a numerical quasiconformal mapping method for the medium parameters identification using applied quasipotential tomography," Mathematical Modeling and Computing, vol. 4 (1), pp. 10-20, 2017.

[5] A. Bomba and M. Boichura, "Spatial analogues of numerical quasiconformal mapping methods for solving identification problems," Zeszyty Naukowe. Telekomunikacja i Elektronika series, vol. 21 (268), pp. 25-34, 2018.

[6] A. Bomba, V. Gavryluk and A. Terebus, "Nonlinear filtration processes mathematical modeling in free boundary spatial mediums," TNTU Bulletin. Mathematical modeling. Mathematics. Physics, vol 16 (4), pp. 168-177, 2011 (in Ukrainian).

[7] A. Ya. Bomba, S. S. Kashtan, D. O. Pryhornytskyi and S. V. Yaroshchak, "Complex Analysis Methods," Rivne: Editorial and Publishing Department of NUWEE, 2013 (in Ukrainian).

[8] S. A. Bochkanov, ALGLIB Reference Manual for C\#. [Online]. Available: https://www.alglib.net/translator/man/manual.csharp.html. [Accessed: May 22, 2020].

[9] “CUDA Toolkit Documentation." [Online]. Available: https://developer.nvidia.com/cuda-zone/. [Accessed: May 22, 2020]. 